\title{
ON REGULAR MATRICES THAT INDUCE THE GIBBS PHENOMENON
}

\author{
JOAQUIN BUSTOZ
}

AbStract. Let $s=\left\{s_{n}(z)\right\}$ be a sequence of complex valued functions defined in a subset $D$ of the complex plane and suppose that $s_{n}(z)$ converges to $f(z)$ for $z \in D$. For $z_{0} \in \bar{D}$ let $K\left(z_{0} ; s\right)$ and $K\left(z_{0} ; f\right)$ be the cores of $s$ and $f$ respectively. We say that $s$ does not have the Gibbs phenomenon at $z_{0}$ if $K\left(z_{0} ; s\right) \subseteq K\left(z_{0} ; f\right)$. The regular matrix $A$ is said to induce the Gibbs phenomenon in $s$ if $K\left(z_{0} ; s\right)$ $\subseteq K\left(z_{0} ; f\right)$ but $K\left(z_{0} ; A s\right) \Phi K\left(z_{0} ; f\right)$. We characterize those regular matrices that induce the Gibbs phenomenon.

1. Let $s=\left\{s_{n}(z)\right\}$ be a sequence of complex valued functions defined in a subset $D$ of the complex plane. Denote the closure of $D$ by $\bar{D}$, and let $z_{0} \in \bar{D}$. Define the sets

$$
G_{n}\left(z_{0} ; s\right)=\left\{w: w=s_{k}(z), k \geqq n, z \in D,\left|z-z_{0}\right|<1 / n\right\} .
$$

Then set $G\left(z_{0} ; s\right)=\bigcap_{n=1}^{\infty} \bar{G}_{n}\left(z_{0} ; s\right)$. It is easy to see that $G\left(z_{0} ; s\right)$ is the set of complex numbers $w$ of the form $w=\lim s_{n(k)}\left(z_{k}\right)$ where $n(1)$ $<n(2)<\cdots$ and $z_{k} \rightarrow z_{0}$. When $s$ is the sequence of partial sums of a real valued Fourier series the set $G\left(z_{0} ; s\right)$ is called the Gibbs set of $s$ at $z_{0}$. We will use the same terminology for $G\left(z_{0} ; s\right)$ when $s$ is any sequence. In the case of Fourier series $G\left(z_{0} ; s\right)$ is connected as a consequence of the Riemann-Lebesgue Theorem. We will show that $G\left(z_{0} ; s\right)$ is connected in the plane under certain conditions on $s$.

Let the closed convex hull of $G_{n}\left(z_{0} ; s\right)$ be denoted by conv $G_{n}\left(z_{0} ; s\right)$, and set $K\left(z_{0} ; s\right)=\bigcap_{n=1}^{\infty} \operatorname{conv} G_{n}\left(z_{0} ; s\right)$. The closed convex set $K\left(z_{0} ; s\right)$ is called the core of $s$ at $z_{0}$. The Gibbs set $G\left(z_{0} ; f\right)$ and core $K\left(z_{0} ; f\right)$ of a function $f(z)$ are the Gibbs set and core of the sequence $f_{n}(z) \equiv f(z)$. $G\left(z_{0} ; f\right)$ is simply the cluster set of $f$ at $z_{0}$. Suppose that $s$ converges to $f(z)$ in $D$. We will say that $s$ does not exhibit the Gibbs phenomenon at $z_{0} \in \bar{D}$ if $K\left(z_{0} ; s\right) \subseteq K\left(z_{0} ; f\right)$. This reduces to the usual definition when $s$ is the partial sum sequence of a Fourier series. In $\S 3$ we will use the core definition to investigate the effect of regular matrix transformation on the Gibbs phenomenon.

The concept of core is due to K. Knopp who defined it for sequences of complex numbers. There is a large body of literature concerning cores of sequences and matrix summability. An account of this is given in [2] along with an extensive bibliography.

Received by the editors June 20, 1969.

AMS Subject Classifications. Primary 4020, 4031; Secondary 4220.

Key Words and Phrases. Core, Gibbs phenomenon, regular matrix, Gibbs set. 
2. In this section we will suppose that $D$ is a locally connected subset of the complex plane. We will consider sequences that satisfy the following condition.

( $\alpha)$ There exists $\left\{\zeta_{k}\right\} \subset D, \zeta_{k} \rightarrow z_{0} \in \bar{D}$, such that

$$
\lim (n \rightarrow \infty)\left[s_{n+1}\left(\zeta_{k}\right)-s_{n}\left(\zeta_{k}\right)\right]=0 \quad \text { for } k=1,2, \cdots .
$$

Lemma. Let $s=\left\{s_{n}(z)\right\}$ be a sequence of continuous functions satisfying $(\alpha)$. Let the nonempty sets $B_{1}$ and $B_{2}$ satisfy the following conditions.

(i) $G\left(z_{0} ; s\right) \subseteq$ int $B_{1} \cup$ int $B_{2}$, where int $B_{i}$ is the interior of $B_{i}$.

(ii) $G\left(z_{0} ; s\right) \cap$ int $B_{i} \neq \varnothing, i=1,2$.

(iii) There exist $\delta>0$ and $N$ such that $s_{n}(z) \in B_{1} \cup B_{2}$ whenever $z \in D$ with $\left|z-z_{0}\right|<\delta$ and $n>N$.

Then $d\left(B_{1}, B_{2}\right)=\inf \left\{\left|w_{1}-w_{2}\right|: w_{i} \in B_{i}\right\}=0$.

Proof. Suppose that $d\left(B_{1}, B_{2}\right)=r>0$. If $s_{n}(w) \in B_{1}$ with $\left|z_{0}-w\right|$ $\leqq \delta$ then $s_{n}(z) \in B_{1}$ for every $z \in D$ with $\left|z-z_{0}\right| \leqq \delta$, since $s_{n}$ is continuous and $B_{1}, B_{2}$ are separated. By condition $(\alpha)$ there exists $\zeta_{i} \in\left\{\zeta_{k}\right\}$ such that $\left|\zeta_{i}-z_{0}\right|<\delta$ and there exists $N$ such that $n \geqq N$ implies $\left|s_{n}\left(\zeta_{i}\right)-s_{n-1}\left(\zeta_{i}\right)\right|<r / 2$. Suppose that $s_{N}\left(\zeta_{i}\right) \in B_{1}$. Since int $B_{2} \cap G\left(z_{0} ; s\right)$ $\neq \varnothing$ there is a first index $M>N$ such that $s_{M}\left(\zeta_{i}\right) \in B_{2}$. Then $s_{M-1}\left(\zeta_{i}\right) \in B_{1}$ and hence $\left|s_{M}\left(\zeta_{i}\right)-s_{M-1}\left(\zeta_{i}\right)\right| \geqq r$. This contradicts the previous inequality and the lemma is proved.

We will say that a set $Q$ is connected in the extended complex plane if in any separation $Q=Q_{1} \cup Q_{2}$, both $Q_{1}$ and $Q_{2}$ are unbounded.

THEOREM 1. Let $s$ be a sequence of continuous functions satisfying condition $(\alpha)$. Then $G\left(z_{0} ; s\right)$ is connected in the extended plane. If $s$ is uniformly bounded in $D$ then $G\left(z_{0} ; s\right)$ is connected.

Proof. We will prove the second statement. The proof of the first is similar and is omitted. Suppose that $G\left(z_{0} ; s\right)$ is not connected. Then there exist nonempty compact sets $Q_{1}$ and $Q_{2}$ such that $G\left(z_{0} ; s\right)=Q_{1} \cup Q_{2}$ and $d\left(Q_{1}, Q_{2}\right)=r_{1}>0$. Further, there exist compact sets $B_{1}$ and $B_{2}$ such that int $B_{i} \supset Q_{i}$ and $0<d\left(B_{1}, B_{2}\right)=r_{2}<r_{1}$. But then $B_{1}$ and $B_{2}$ satisfy the conditions of the lemma, and thus $d\left(B_{1}, B_{2}\right)=0$. This contradiction establishes the result.

If $s$ is real valued it is not necessary to distinguish between the bounded and unbounded case. It is perhaps worthwhile to state this as

THEOREM 2. Let $s$ be a sequence of continuous real functions that satisfy $(\alpha)$. Then $G\left(z_{0} ; s\right)$ is connected. 
The sequence $\left\{e^{i n}\right\}$ shows that $(\alpha)$ is not necessary for connectedness. Examples of sequences that satisfy $(\alpha)$ are sequences converging in $D$, and partial sums of Fourier series.

3. Let $A=\left(a_{n k}\right)$ be a regular summability matrix. We will write $t=A s$ for the transform sequence $t_{n}(z)=\sum a_{n k} s_{k}(z)$. In this section we will consider the following situation. Suppose that $s=\left\{s_{n}(z)\right\}$ converges pointwise to $f(z)$ in $D$. Suppose further that at $z_{0} \in \bar{D}, K\left(z_{0} ; s\right)$ $\subseteq K\left(z_{0} ; f\right)$, so that $s$ does not have the Gibbs phenomenon at $z$. Is it possible for $A s$ to show the phenomenon at $z_{0}$ even though $s$ does not? We will show that this is indeed possible, and when this occurs we will say that $A$ induces the Gibbs phenomenon in $s$. We will use the notation $\|A\|=\lim \sup (n \rightarrow \infty) \sum_{k}\left|a_{n k}\right|$.

Theorem 3. The regular matrix $A$ induces the Gibbs phenomenon in some uniformly bounded sequence if and only if $\|A\|>1$.

Proof. Since $A$ is regular, $\|A\| \geqq 1$. Suppose that $\|A\|=1$, and that $s$ is a uniformly bounded sequence converging to $f$ with $K\left(z_{0} ; s\right)$ $\subseteq K\left(z_{0} ; f\right)$. Since $\|A\|=1$ the Bounded Core Theorem [1] implies $K\left(z_{0} ; A s\right) \subseteq K\left(z_{0} ; s\right)$. Hence $K\left(z_{0} ; A s\right) \subseteq K\left(z_{0} ; f\right)$ and $A$ does not induce the Gibbs phenomenon. To prove the theorem in the opposite direction we will show that if $\|A\|>1$ then it is possible to construct a sequence with the desired property. The proof is an adaptation of a proof due to W. Hurwitz [3]. If $\|A\|>1$ then there exist $\lambda>0$ and sequences of natural numbers $\{n(p)\},\{N(p)\}$ such that for $p=1$, $2, \cdots$ :

$$
\sum_{k=1}^{N(p)}\left|a_{n(p), k}\right|>1+4 \lambda, \quad \sum_{k=1}^{N(p)-1}\left|a_{n(p), k}\right|<\lambda, \quad \sum_{k=N(p)+1}^{\infty}\left|a_{n(p), k}\right|<\lambda .
$$

For the purpose of the construction we assume that the complement of $\{N(p)\}$ in the natural numbers has the cardinality of the natural numbers. This assumption does not result in any loss of generality. Let $f(x)=\exp i / x, x \neq 0,-1 \leqq x \leqq 1$ and $f(0)=0$.

Then $K(0, f)$ is the closed unit disc. Now define the sequence $s=\left\{s_{k}(x)\right\}$ as follows. We will write $x_{p}=1 / n(p)$.

$$
\begin{aligned}
s_{k}(0) & =0, \quad k=1,2, \cdots, \\
s_{k}(x) & =\exp i / x \quad \text { if } x \neq 0 \text { and } x \neq 1 / N(p), \quad k=1,2, \cdots, \\
s_{k}\left(x_{p}\right) & =\exp i N(p), \quad k \leqq N(p-1), \\
& =(-1)^{p} \exp \left(-i \arg a_{n(p), k}\right), \quad N(p-1)<k \leqq N(p), \\
& =\exp i N(p), \quad k>N(p) .
\end{aligned}
$$


Then $s_{n}(x) \rightarrow f(x)$ and $K(0 ; s)$ is the closed unit disc. Hence $s$ does not exhibit the Gibbs phenomenon. Now

$$
\begin{aligned}
t_{n(p)}\left(x_{p}\right)= & \sum_{k} a_{n(p), k} s_{k}\left(x_{p}\right) \\
= & \sum_{k=1}^{N(p-1)} a_{n(p), k} s_{k}\left(x_{p}\right)+\sum_{k=N(p-1)+1}^{N(p)} a_{n(p), k} s_{k}\left(x_{p}\right) \\
& +\sum_{k=N(p)+1}^{\infty} a_{n(p), k} s_{k}\left(x_{p}\right) \\
= & \exp i N(p) \sum_{k=1}^{N(p-1)} a_{n(p), k}-(-1)^{p} \sum_{k=1}^{N(p-1)}\left|a_{n(p), k}\right| \\
& +(-1)^{p} \sum_{k=1}^{N(p)}\left|a_{n(p), k}\right|+\exp i N(p) \sum_{k=N(p)+1}^{\infty} a_{n(p), k} .
\end{aligned}
$$

Taking real parts:

$$
\begin{aligned}
(-1)^{p} \operatorname{Re} t_{n(p)}\left(x_{p}\right)= & \sum_{k=1}^{N(p)}\left|a_{n(p), k}\right|-\sum_{k=1}^{N(p-1)}\left|a_{n(p), k}\right| \\
& +(-1)^{p} \operatorname{Re} \exp i N(p) \sum_{k=1}^{N(p-1)} a_{n(p), k} \\
& +(-1)^{p} \operatorname{Re} \exp i N(p) \sum_{k=N(p)+1}^{\infty} a_{n(p) k} \\
\geqq & \sum_{k=1}^{N(p)}\left|a_{n(p), k}\right|-2 \sum_{k=1}^{N(p-1)}\left|a_{n(\rho) k}\right| \\
& -\sum_{k=N(p)+1}^{\infty}\left|a_{n(p), k}\right| \\
> & 1+4 \lambda-2 \lambda-\lambda=1+\lambda .
\end{aligned}
$$

Now $(-1) p \operatorname{Re} t_{n(p)}\left(x_{p}\right)>1+\lambda$ implies that $K\left(z_{0} ; A s\right)$ must have points lying outside the unit circle. Hence $K(0 ; f)$ does not contain $K(0 ; A s)$ and $A$ induces the Gibbs phenomenon in $s$.

It is possible to prove a theorem similar to Theorem 3 that holds for sequences of bounded functions. We must however restrict ourselves to row-finite regular matrices. The following terminology will be usid. The row-finite matrix $A$ will be said to be essentially real and nonnegative if the elements of $A$ are real and nonnegative except possibly for finitely many rows or columns. A preliminary result will be required. This next theorem is an extension of the unbounded 
core theorem for sequences of complex numbers [2, p. 148]. The proof is similar to that given in the reference and is omitted.

THEOREM 4. Let $A$ be row-finite and regular. Then $K\left(z_{0} ; A s\right)$ $\subseteq K\left(z_{0} ; s\right)$ for every sequence of bounded functions if and only if $A$ is essentially real and nonnegative.

Theorem 5. Let $A$ be row-finite and regular. Then $A$ does not induce the Gibbs Phenomenon in any sequence of bounded functions if and only if $A$ is essentially real and nonnegative.

Proof. Let $s_{n}(z) \rightarrow f(z)$ and $K\left(z_{0} ; s\right) \subseteq K\left(z_{0} ; f\right)$. If $A$ is essentially real and nonnegative then $K\left(z_{0} ; A s\right) \subseteq K\left(z_{0} ; f\right)$ by Theorem 4 . Hence $A$ does not induce the Gibbs phenomenon. To prove the converse suppose first that $a_{n(p), k(p)}<0$ where $n(1)<n(2)<\cdots$ and $k(1)<k(2)$ $<\cdots$. Let $f(x)=1 / x$ for $0<x \leqq 1$ and $f(0)=0$. Set $x_{p}=1 / n(p)$. If $k \notin\{k(p)\}$ set $s_{k}(x)=1 / x$ for $1 / k \leqq x \leqq 1$ and $s_{k}(x)=0$ for $0 \leqq x<1 / k$. Set $s_{k(p)}\left(x_{p}\right)=-1 / a_{n(p), k(p)} ; s_{k(p)}(x)=0$ for $0 \leqq x<x_{p}$ and $s_{k(p)}(x)=1 / x$ for $x_{p}<x \leqq 1$. Then $s=\left\{s_{k}(x)\right\}$ is a sequence of bounded functions converging to $f$, and $K(0, s)=K(0, f)=[0, \infty)$. But $t_{n(p)}\left(x_{p}\right)=-1$ and hence $K(0, A s)$ is not contained in $K(0, f)$.Thus $A$ induces the Gibbs phenomenon. On the other hand, suppose that $\operatorname{Im} a_{n(p), k(p)} \neq 0$ for $p=1,2, \cdots$. Say that $\operatorname{Im} a_{n(p), k(p)}<0$. Let $f(x)=i / x$ for $0<x \leqq 1$ and $f(0)=0$. If $k \notin\{k(p)\}$ then set $s_{k}(x)=i / x$ for $1 / k \leqq x \leqq 1 ; s_{k}(x)=0$ for $0 \leqq x<1 / k$. Also set $s_{k(p)}\left(x_{p}\right)=i / \mathrm{Im} \quad a_{n(p), k(p)} ; s_{k(p)}(x)=0$ if $0 \leqq x<x_{p}$, and $s_{k(p)}(x)=i / x$ if $x_{p}<x \leqq 1$. Again $K(0, s)=K(0, f)$ each set consisting of the imaginary axis $i y, y \geqq 0$. But $\operatorname{Re} t_{n(p)}\left(x_{p}\right)=-1$ and hence $K(0, A s)$ is not contained in $K(0, f)$. This completes the proof.

4. It is of interest to ask to what extent Theorems 3 and 5 apply to Fourier series. Clearly if $\|A\|=1$ then $A$ cannot induce the Gibbs phenomenon in uniformly bounded Fourier series. Also if $A$ is essentially real and nonnegative, then $A$ cannot induce the phenomenon in any Fourier series. But when $A$ does not satisfy either of the above conditions there does not necessarily exist a Fourier sequence in which $A$ induces the Gibbs phenomenon. Thus we can ask the following question: Does there exist a regular matrix $A$ and a Fourier series in which $A$ induces the Gibbs phenomenon?

We will answer this question affirmatively by proving a more genera 1 result. Previously we have considered $G\left(z_{0} ; s\right)$ with $z_{0} \in \bar{D}$ where $D$ is the domain of definition of $s$. In this section we will take $z_{0} \in D$ so that each $s_{n}$ is defined at $z_{0}$. 
THEOREM 6. Let $s=\left\{s_{n}(z)\right\}$ be a sequence of functions continuous and uniformly bounded in $D$. Let $z_{0} \in D$ and let $G\left(z_{0} ; s\right)$ contain more than one point. Then for every point w there exists a regular matrix $A$ such that $w \in G\left(z_{0} ; A s\right)$.

Proof. We may take $z_{0}=0$. Our proof is based on the assertion that under the given hypothesis there exists a sequence $\left\{z_{p}\right\} \subset D$, $z_{p} \rightarrow 0$, and increasing sequences of natural numbers $\{n(p)\},\{m(p)\}$ with $n(p) \neq m(p)$, such that $s_{m(p)}\left(z_{p}\right) \rightarrow w_{1}$ and $s_{n(p)}\left(z_{p}\right) \rightarrow w_{2}$ with $w_{1} \neq w_{2}$. The assertion is clearly true if $s_{n}(0)$ diverges.

Suppose then that $s_{n}(0)$ converges, say $s_{n}(0) \rightarrow w_{1}$. Since $G(0 ; s)$ contains at least two points there exists $w_{2} \neq w_{1}, z_{p} \rightarrow 0$, and $m(p)$ such that $s_{m(p)}\left(z_{p}\right) \rightarrow w_{2}$. Since the $s_{n}$ are continuous there exists a nested sequence of discs $R_{n}=\left\{z:|z|<\delta_{n}\right\}, \delta_{n} \rightarrow 0$, such that $z \in R_{n}$ implies $\left|s_{n}(0)-s_{n}(z)\right|<1 / n$. We may assume that each $z_{p}$ belongs to some $R_{n}$. For each $p$ let $R_{n(p)}$ be the smallest disc such that $z_{p} \in R_{n(p)}$. We may assume that the $n(p)$ are distinct (if not then we can choose a subsequence of $\left\{z_{p}\right\}$ for which this will be true). There are at most finitely many $p$ such that $z_{p} \in R_{m(p)}$. For if $z_{p} \in R_{m(p)}$ for infinitely many $p$ then by definition of $R_{m(p)}$ we would have $\left|s_{m(p)}\left(z_{p}\right)-s_{m(p)}(0)\right|$ $<1 / m(p)$ and this implies that $w_{1}$ is a limit point of $s_{m(p)}\left(z_{p}\right)$ which is impossible. Thus $m(p)=n(p)$ for at most finitely many $p$. By discarding this finite number of indices we may assume that $m(p) \neq n(p)$, $p=1,2, \cdots$. We also have that $\left|s_{n(p)}(0)-s_{n(p)}\left(z_{p}\right)\right|<1 / n(p)$ and hence $s_{n(p)}\left(z_{p}\right) \rightarrow w_{1}$.

We now know that there exist sequences such that $s_{n(p)}\left(z_{p}\right) \rightarrow w_{1}$, $s_{m(p)}\left(z_{p}\right) \rightarrow w_{2}$ with $w_{1}, w_{2} \in G(0 ; s), w_{1} \neq w_{2}$, and $n(p) \neq m(p)$. Given $w € G(0 ; s)$ set $\alpha=\left(w-w_{2}\right) /\left(w_{1}-w_{2}\right)$. Define the regular matrix $A$ by $a(k, n(k))=\alpha, a(k, m(k))=1-\alpha, a(k, j)=0$ elsewhere. Then if $\left\{t_{p}\right\}$ is the $A$-transform of $s$, we have $t_{p}\left(z_{p}\right)=\alpha s_{n(p)}\left(z_{p}\right)+(1-\alpha) s_{m(p)}\left(z_{p}\right)$ and hence $t_{p}\left(z_{p}\right) \rightarrow \alpha w_{1}+(1-\alpha) w_{2}=w$. This completes the proof.

As a consequence of this last result we have the following

Theorem 7. Let $s=\left\{s_{n}(z)\right\}$ be a sequence of functions continuous and uniformly bounded in $D$, and let $s_{n}(z) \rightarrow f(z)$ in $D$. Suppose that $z_{0} \in D$, that $K\left(z_{0} ; s\right) \subseteq K\left(z_{0} ; f\right)$, and that $K\left(z_{0} ; s\right)$ contains more than one point. Then there exists a regular matrix $A$ such that $A$ induces the Gibbs phenomenon in s.

Proof. We need only choose $w \notin K\left(z_{0} ; f\right)$ and Theorem 6 assures us that there exists $A$ such that $w \in G\left(z_{0} ; A s\right) \subseteq K\left(z_{0} ; A s\right)$.

We can now answer the question posed at the beginning of this section. Izumi and Satô [4] have shown that there exists a function $f$ 
with a bounded discontinuity at $x=0$ such that the Fourier series of $f$ does not have the Gibbs phenomenon at $x=0$. The Gibbs set $G(0, s)$ of this Fourier series contains more than one point. Thus by Theorems 6 and 7 we have

THEOREM 8. There exists a regular matrix $A$ and a function $f$ such that $A$ induces the Gibbs phenomenon in the Fourier series of $f$.

Finally then we may ask: What are necessary and sufficient conditions on a regular matrix $A$ so that $A$ does not induce the Gibbs phenomenon in Fourier series?

\section{REFERENCES}

1. R. Atalla and J. Bustoz, On sequential cores and a theorem of R. R. Phelps, Proc. Amer. Math. Soc. 21 (1969), 36-42.

2. R. G. Cooke, Infinite matrices and sequence spaces, Macmillan, New York, 1950; reprint, Dover, New York, 1955. MR 12, 694.

3. W. A. Hurwitz, Some properties of methods of evaluation of divergent sequences. Proc. London Math. Soc. (2) 26 (1927), 231-248.

4. S. Izumi and M. Satô, Fourier series. X: Rogosinski's Lemma, Ködai Math. Sem. Rep. 8 (1956), 164-180. MR 19, 138.

University of Cincinnati, Cincinnati, Ohio 45221 